\title{
ON PSEUDO-ANALYTIC FUNCTIONS
}

\author{
D. A. STORVICK
}

1. Introduction. Many of the properties of analytic functions can be proved in a purely topological manner, so that such properties are then valid for the larger class of functions which are topologically equivalent to analytic functions. The importance of such functions has been recognized fairly recently, particularly in the theory of partial differential equations, where certain solutions have been shown to possess the topological properties of analytic functions, i.e., interiority and continuity, but not necessarily the analytic properties of complex differentiability and integrability.

We shall assume in what follows that $w=f(z)=u(x, y)+i v(x, y)$ is an interior transformation in the sense of Stoilow ${ }^{11}$ in an arbitrary domain, $D, i_{0} \mathrm{e}$, $w=f(z)$ is continuous and single-valued in $D$, takes open sets in $D$ into open sets in the $w$-plane, and does not take any continuum in $D$ into a single point of the $w$-plane.

If, in addition,

i) the first partial derivatives, $\imath_{x}, \imath t y, v_{x}, v_{y}$ exist and are continuous $n$,

ii) the Jacobian $f(f) \equiv\left|\begin{array}{lll}u_{x} & u_{y} \\ v_{x} & v_{y}\end{array}\right|$ is positive at every point of $D$, except for at most a countable set, the function $w=f(z)$ will be called pseutoanalytic in $D$. If $f(z)$ is pseudo-analytic in some neighborhood of a point $z_{0}$, we shall say that $f(z)$ is pseudo-analytic at $z_{0}$.

An infinitesimal circle with center at $z_{0}$ is mapped by $w=f(z)$ into an infinitesimal ellipse with center at $w_{0}=f\left(z_{0}\right)$. Corresponding to an element $\mid$ wa $\mid$ of arc in the $z$-plane, there will be an element of arc $|d w|$ in the w-plane. We have that $|d w|^{2}=E d x^{2}+2 F d x d y+G d y^{2}$ where

$$
\begin{aligned}
& E=u_{x}^{2}+v_{x}^{2}, \\
& F=u_{x} u_{y}+v_{x} v_{y}, \\
& G=u_{y}^{2}+v_{y}^{2} .
\end{aligned}
$$

Received April 8, 1.957.

1) See Stoillow [11]. 
At $z_{0}=x_{0}+i y_{0}$, if $|d z|=$ constant, the maximum and minimum values of $|d w|^{2}$ will give us the major and minor axes, $a$ and $b$ respectively, of the infinitesimal ellipse mentioned above. The dilatation-quotient $Q\left[f\left(z_{0}\right)\right]$ of $f(z)$ at $z=z_{0}$ is defined as the ratio of the major and minor axes $\frac{a}{b}$, and

$$
Q[f(z)]=\frac{E+G+\left[(E+G)^{2}-4\left(E G-F^{2}\right)\right]^{1 / 2}}{2\left[E G-F^{2}\right]^{1 / 2}} .
$$

Because $w=f(z)$ is an interior transformation, it possesses the maximummodulus principle and, if non-zero, the minimum-modulus principle. The composition of two pseudo-analytic functions is pseudo-analytic and its dilatationquotient is bounded by the product of their respective dilatation-quotients.

If $f(z)$ is analytic, then $Q[f(z)]$ is identically 1 , so that the dilatationquotient is a conformal invariant; we also observe that for the inverse function $z=f^{-1}(w)$ of $w=f(z), Q[f(z)]=Q\left[f^{-1}(w)\right]$.

In the remainder of this paper we shall assume that $D$ is the unit circle $|z|<1$, and that $Q[f(z)]$ is uniformly bounded in $|z|<1, Q \leqq M$. The latter assumption implies the existence of a finite constant $K \geqslant 1$ such that $E+G$ $\leqq 2 K\left[E G-F^{2}\right]^{1 / 2}$. We state first a well-known theorem on the correspondence of boundaries under univalent pseudo-analytic functions which we shall refer to as quasi-conformal mappings.

THEOREM 1. Let $w=f(z)$ be a univalent pseudo-analytic function mapping $|z|<1$ onto $|w|<1$, and let $Q[f(z)] \leqq M$. Then $w=f(z)$ can be extended to give a one-to-one bicontinuous transformation of $|z| \leqq 1$ onto $|w| \leqq 1$.

Several authors including Hössjer, Frostman, Lohwater, Nevanlinna and Seidel have discussed the class of functions $w=f(z)$, meromorphic in $|z|<1$ such that the modulus $\left|f\left(r e^{i \theta}\right)\right|$ has radial limit 1 for all $e^{i \theta}$ on $|z|=1$ except for a possible set of values on $|z|=1$ of linear measure zero. This class of functions is often called class $(U)$. The subclass of functions of bounded characteristic is called class $(B)$ and the subclass of bounded analytic functions class $(A)$. The purpose of this note is to extend the notion of class $(A)$ (and class $(U))$ to pseudo-analytic functions. The need for this has been pointed out by Noshiro in his paper: On the theory of cluster sets of analytic functions, Sugaku 5 no. 2 (1953) 65-72, (Japanese), which will appear shortly in English in a translation by the American Mathematical Society. 
We remark first that Pfluger [9, p. 130] has shown that, under the quasiconformal mapping of $|z|<1$ onto $|w|<1$ of Theorem 1 , a set $E_{z}$ on $|z|=1$ of capacity zero ${ }^{2)}$ is transformed into a set $E_{w}$ on $|w|=1$ of capacity zero.

Definition. A function $f(z)$, pseudo-analytic and of bounded dilatationquotient in $\mid z !<1$, will be called of class $\left(U^{*}\right)$ in $|z|<1$, if $\lim _{r \rightarrow 1}\left|f\left(r e^{i \theta}\right)\right|=1$ for all $e^{i \theta}$ on $|z|=1$, except for a possible set of logarithmic capacity zero. A function $f(z)$ of class $\left(U^{*}\right)$ will be called of class $\left(A^{*}\right)$ in $|z|<1$ if $|f(z)|<1$.

We remark that, even for functions of class $\left(A^{*}\right)$, no analogues of the Fatou or Riesz Theorem are valid which involve exceptional sets of measure zero. Indeed, a recent example of Beurling and Ahlfors [Acta Math. 96 (1956), 125-142] shows that such theorems will not hold, in general, so that, in a sense, the class $\left(A^{*}\right)$ considered in this paper is the most general extension of the class $(A)$ to the class of pseudo-analytic functions.

However, there is the following generalized maximum pinciple first proved by Noshiro [7], involving an exceptional set of capacity zero, for which we shall give an alternate proof which we feel is more elementary in the sense that it is independent of the deeper Star Theorem of Gross. The example cited in the last paragraph shows, too, that Theorem 2 is the most general form of an extended maximum principle which can be expected for pseudo-analytic functions.

Theorem 2. Let $w=f(z)$ be pseudo-analytic and bounded, $|f(z)|<M$, in $|z|<1$. If the $\lim _{z \rightarrow \zeta} \sup |f(z)| \leqq m<M$ for all points $\zeta$ of $|z|=1$ except for $a$ possible set $E$ of capacity zero on $|z|=1$, then $|f(z)|<m$ in $|z|<1$.

Proof. Because the Riemann surface $W$ of the inverse function $f^{-1}(w)=z$ is of hyperbolic type, let $t=F^{-1}(w)$ be the function which maps $W$ one-to-one conformally onto $|t|<1$. The inverse function $w=F(t)$ is analytic in $|t|<1$. Setting $t=F^{-1}(w)=F^{-1}(f(z)) \equiv \phi(z)$, we see that $t=\phi(z)$ is a univalent pseudoanalytic function giving a quasi-conformal mapping of $|z|<1$ onto $|t|<1$. Let $E_{t}$ be the image of $E$ under $t=\phi(z)$. By Pfluger's Theorem [9, p. 130], we know that the capacity of $E_{t}$ is zero.

Since $w=F(t)=f\left(\phi^{-1}(t)\right)$, we see that $|f(t)|<M$ in $|t|<1$, and for $\tau E E_{t}, \lim _{t \rightarrow:} \sup |F(t)|=\lim _{t \rightarrow \tau} \sup \left|f\left(\phi^{-1}(t)\right)\right|=\lim _{z \rightarrow \zeta=\phi_{i \tau}} \sup _{i}|f(z)| \leqq m<M$ and $\zeta \notin E$.

2) See Nevanlinna [6; pp. 112 f.]. 
By the Poisson integral representation of a bounded analytic function in $|t|<1$, we can conclude that $|F(t)|<m$ in $|t|<1$. Since $F(t)=f\left(\phi^{-1}(t)\right)$, we see that $|f(z)|<m$ in $|z|<1$.

We now prove the main result of this note, which is a modification of Theorem 5 of [3].

THEOREM 3. Let $w=f(z)$ be pseudo-analytic of class $\left(U^{*}\right)$ in $|z|<1$ and omit the value zero. Then, unless $f(z)$ is identically constant, $f(z)$ must admit either 0 or $\infty$ as an asymptotic value.

We shall suppose that $f(z)$ is not identically constant in $|z|<1$ and that $f(z)$ takes on values of modulus less than 1 . If $|f(z)|>1$, we shall consider $1 / f(z)$ which is of class $\left(A^{*}\right)$ and omits the value zero and proceed with the proof. It follows from Theorem 2 that the open sets

$$
\theta_{n}=\left\{z|| z|<1 ;| f(z) \mid<\frac{1}{n}\right\}
$$

are non-empty for all positive integers $n$. Let us fix $n$ and consider an arbitrary connected component $G_{k}^{(n)}$ of $\theta_{n}$. It is an immediate consequence of the maximum and minimum modulus principles for $f(z)$ that $G_{k}^{(n)}$ is simply-connected and that the intersection $E_{k}^{(n)}$ of the frontier of $G_{k}^{(n)}$ with $|z|=1$ is not empty.

We show next that the frontier $\operatorname{Fr}\left(G_{k}^{(n)}\right)$ of $G_{k}^{(n)}$ is locally connected. Now that part of $\operatorname{Fr}\left(G_{k}^{(n)}\right)$ lying inside $|z|<1$ consists of a piecewise smooth curve, being the locus of points where $|f(z)|=\frac{1}{n}$. If $P$ is a point of $E_{k}^{(n)}$, and if $\operatorname{Fr}\left(G_{k}^{(n)}\right)$ is not locally connected at $P$, then, by a well-known theorem [13; p. 19], there exists a non-degenerate subcontinuum $H$ of $\operatorname{Fr}\left(G_{k}^{(n)}\right)$ containing $P$ and such that $\operatorname{Fr}\left(G_{k}^{(n)}\right)$ is not locally connected at any point of $H$. Since $H$ must lie on $|z|=1$, it follows that $H$ is an arc of $|z|=1$. Moreover, there exist [13; p. 18] a circular neighborhood $V(P)$ of $P$ and a sequence of mutually disjoint components $N_{2}, N_{2}, \ldots$ of $\operatorname{Fr}\left(G_{k}^{(n)}\right) \cap \overline{V(P)}$ converging to a nondegenerate limiting arc $N \subset H$ containing $P$. If $R$ is a circular neighborhood about the midpoint of $N$, of radius less than one-fourth the length of $N$, it follows that a radius of $|z|<1$ drawn to any point $Q$ of that sub-arc $N_{0}$ cut out of $N$ by $R$ must cross the components $N_{j}$ of $\operatorname{Fr}\left(G_{k}^{(n)}\right) \cap \overline{V(P)}$ arbitrarily close to $Q$. Along such a radius of $|z|<1$, if $\left|f\left(r e^{i \theta}\right)\right|$ tends to a limit, this limit must be $1 / n$, since $|f(z)|=\frac{1}{n}$ at the points where such a radius inter- 
sects the $N_{j}$. Since this happens at every point of $N_{0}$, we have contradicted the hypothesis that $\left|f\left(r e^{i \theta}\right)\right| \rightarrow 1$ on all radii except possibly those radii terminating in a set of capacity zero on $|z|=1$. Hence $\operatorname{Fr}\left(G_{k}^{(n)}\right)$ is locally connected.

We now prove that the set $E_{k}^{(n)}$ on $|z|=1$ has logarithricic capacity zero. We denote by $E$ the set of points of $|z|=1$ for which $\lim _{r \rightarrow 1}\left|f\left(r e^{i 0}\right)\right|=1$; by definition the capacity of $\widetilde{E}$ is zero, where $\widetilde{E}$ denotes the complement of $E$ on $|z|=1$. Because of the decomposition $E_{k}^{(n)}=\left(E_{k}^{(n)} \cap E\right) \cup\left(E_{k}^{(n)} \cap \widetilde{E}\right)$, it will suffice to remark that $E_{k}^{(n)} \cap E$ has logarithmic capacity zero, Indeed, by a recent result of Bagemihl (see Theorem 2 and Corollary I of $[1 ;$ p. 380]), which extends an idea in the proof of Theorem 5 of [3], it follows that $E_{k}^{(n)} \cap E$ is at most enumerable, and consequently has logarithmic capacity zero.

To complete the proof of Theorem 3 , we construct a nesting sequence of components as follows: In $G_{k}^{(n)}$ there exists at least one component say $G_{k_{2}}^{(n+1)}$ of $\theta_{n+1}$, in $G_{k_{1}}^{(n+1)}$ there exists at least one component $G_{k_{2}}^{(n+2)}$, etc., so that

$$
G_{k}^{(n)} \supset G_{k_{1}}^{(n+1)} \supset G_{k_{2}}^{(n+2)} \supset \ldots
$$

Otherwise, for some fixed integer $p(p=0,1,2, \ldots)$, the sequence (2) must terminate $G_{k}^{(n)} \supset G_{k_{1}}^{(n+1)} \supset \ldots \supset G_{k_{p}}^{(n+p)}$, with $G_{k_{p}}^{(n+p)} \neq \phi$ and with no component of $\theta_{n+p+1}$ inside $G_{k p}^{(n+p)}$.

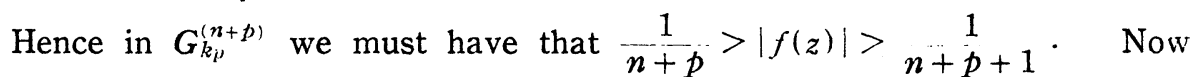
at all points of the frontier of the region $G_{k_{x}}^{(n+\not)}$, with the possible exception of the closed set $E_{k p}^{(n+p)}$ of capacity zero, we have $\lim \inf |f(z)|=\frac{1}{n+p}$ with $|f(z)|>\frac{1}{n+p+1}$ everywhere in $G_{k p}^{(n+p)}$.

A simple modification of Theorem 3 shows that we must have $|f(z)|$ $>\frac{1}{n+p}$ in $G_{k p}^{(n+p)}$, which contradicts the structure of that region. Hence the sequence (2) cannot terminate.

We may now choose a sequence of points $\left\{z_{p}\right\}, z_{p} \in G_{k_{p}}^{(n+p)}$ and form a simple arc (or polygonal path) connecting $z_{1}$ with $z_{p}$ which lies in the nested sequence of components. The intersection $\bigcap_{p} G_{k_{p}}^{(n+p)}$ must consist of a single point of $|z|=1$; for, otherwise, this intersection must contain a continuum joining any two points of the intersection. If this continuum lies on $|z|=1$, we would then contradict the hypothesis that $\left|f\left(r e^{i \theta}\right)\right| \rightarrow 1$ on all radii except possibly those terminating in a set of capacity zero on $|z|=1$, while if part of 
this continuum lies in $|z|<1$, we contradict the assumption that $f(z) \neq 0$ in $|z|<1$. Thus we have constructed an arc terminating at a point $e^{i \theta_{0}}$ of $|z|=1$ such that, as $z \rightarrow e^{i \theta_{0}}$ along $L, f(z) \rightarrow 0$. Theorem 3 is then proved.

Since a function of class $\left(A^{*}\right)$ is of class $\left(U^{*}\right)$, it follows that we have as a corollary that if a non-constant function $f(z)$ of class $\left(A^{*}\right)$ omits the value zero, then $f(z)$ must admit zero as an asymptotic value. However, since the composition of two pseudo-analytic functions is pseudo-analytic, we can prove a more precise theorem for the class $\left(A^{*}\right)$.

TheOREm 4. Let $f(z)$ be of class $\left(A^{*}\right)$ in $|z|<1$ and omit the value $\alpha$, $|\alpha|<1$. Then unless $f(z)$ is identically constant, $f(z)$ must admit $\alpha$ as an asymptotic value.

Indeed, the function $g(z)=\frac{f(z)-\alpha}{1-\bar{\alpha} f(z)}$ is pseudo-analytic of class $\left(A^{*}\right)$ is $|z|<1$ and omits the value 0 . Hence $g(z)$ must admit the value 0 as an asymptotic value, by Theorem 3. Thus $\alpha$ is an asymptotic value of $f(z)$. From Theorem 5 below we can also conclude that $\alpha$ is a radial limit value.

We next extend to pseudo-analytic functions a well-known theorem of Lindelöf (see, Sur un principe général de l'Analyse et ses applications á la théorie de la représentation conforme, Acta Soc. Scient. Fennicae 46, No. 4 (1915), p. 10) for general analytic functions, rather than for functions of class $(A)$ or class $(U)$.

THEOREM 5. Let $w=f(z)$ be a bounded pseudo-analytic function in $|z|<1$ and let $L$ be a Jordan arc lying in $|z|<1$ except for one of its end points $e^{i 0_{0}}$. If $\lim f(z)=\alpha$ as $z \rightarrow e^{i 0_{0}}$ along $L, \lim f(z)=\alpha$ exists uniformly in every Stoltz angle with vertex at $e^{i \theta_{0}}, \arg \left|1-z e^{-i \theta_{0}}\right| \leqq \frac{\pi}{2}-\delta, \frac{\pi}{2}>\delta>0$.

Proof. Let $t=F^{-1}(w)$ be the function which maps the Riemann surface of the inverse function of $w=f(z)$, one-to-one conformally onto $|t|<1$. The function $t=F^{-1}(f(z)) \equiv \phi(z)$ gives a quasi-conformal mapping of $|z|<1$ onto $|t|<1$ and by Theorem 1 above, $\phi(z)$ can be extended to give a homeomorphism of $|z| \leqq 1$ onto $|t| \leqq 1$. By a recent result of Jenkins (see Lemma 1, [2], p. 344), it follows that if $M$ is arc in $|z| \leqq 1$ having one endpoint at $e^{i \theta_{0}}$ on $|z|=1$, otherwise lying in $|z|<1$ and tending to $e^{i n_{0}}$ non-tangentially, i.e., so as to lie in the angle between two chords of $|z|=1$ each with one endpoint at $e^{i \theta_{0}}$, (a Stoltz angle) then its image $U=\phi(M)$ under $t=\phi(z)$ tends to $e^{i \gamma}=\phi\left(e^{i \theta_{0}}\right)$ 
non-tangentially. Since $t=\phi(z)$ is a homeomorphism, $\lambda=\phi(L)$ also terminates at $e^{i:}$ on $|t|=1$.

Now $F(t) \rightarrow \alpha$ as $t \rightarrow e^{i \pi}$ along $\lambda$ since $F(t)=F(\phi(z))=F\left(F^{-1}(f(z))\right)$ $=f(z)$ and $f(z) \rightarrow \alpha$ as $z \rightarrow e^{i \theta_{0}}$ along $L$. Since $w=F(t)$ is a bounded analytic function in $|t|<1$, it follows from Lindelöf's theorem [6; p. 70] that $\lim F(t)$ $=\alpha$ uniformly in any Stoltz angle with vertex at $t=e^{i \text { r }}$. Hence $F(t) \rightarrow \alpha$ as $t \rightarrow e^{i r}$ along $U$ and thus $\lim f(z)=\alpha$ as $z \rightarrow e^{i \theta_{0}}$ along $M$.

It is well known ${ }^{3 /}$ that an analytic function of class $(A)$ assumes all values of $|z|<1$ except for a possible set of capacity zero. We shall show that an analogous theorem is valid for class $\left(A^{*}\right)$.

THEOREM 6. Let $w=f(z)$ be a non-constant pseudo-analytic function of class $\left(A^{*}\right)$ in $|z|<1$. Then the set of values $w$ in $|w|<1$ such that the equation $f(z)-w=0$ has no solution in $|z|<1$ is of capacity zero.

Let $S$ denote the set of values $w$ for which the equation $f(z)-w=0$ has no solution in $|z|<1$. By Theorem 4 each point of $S$ is an asymptotic value of $f(z)$ in $|z|<1$, so that each point of $S$ is arcwise accessible from the interior of the domain $G$ onto which $f(z)$ maps $|z|<1$. If we map the (necessarily hyperbolic) Riemann surface $R_{i}$ of $f(z)$ conformally onto $: t<1$ by means of an analytic function $t=F^{-1}(w)$, the composed function $t=F^{-1}(f(z))$ is a univalent pseudo-analytic function in $|z|<1$ which gives a quasi-conformal mapping of $|z|<1$ onto $|t|<1$. By Theorem 1, the function $t=F^{-1}(f(z))$ $\equiv \phi(z)$ can be extended so as to give a homeomorphism of $|z|=1$ onto $|t|=1$. Denote by $E_{z}$ the set of points of $|z|=1$ for which $\lim _{r \rightarrow 1}\left|f\left(r e^{i 0}\right)\right|=1$ and by $\widetilde{E}_{z}$ the complement of $E_{z}$. By definition, the capacity of $\widetilde{E}_{z}$ is zero. Denote by $E_{t}$ and $\widetilde{E}_{t}$ the sets of points on $|t|=1$ which, under the homeomorphism $t=\phi(z)$, correspond to $E_{z}$ and $\widetilde{E}_{z}$ respectively. By Pfluger's theorem [9], the capacity of $E_{t}$ is zero and by Fatou's theorem [6; p. 206], the bounded analytic function $v=F(t)$ has radial limit values $F\left(e^{i \eta}\right)=\lim _{r \rightarrow 1} F\left(r e^{i \theta}\right)$ for all $e^{i \theta}$ except at most a set of measure zero. Denote by $(i)$ the set of $e^{i \theta}$ such that $\left|F\left(e^{i \theta}\right)\right|<1$. We remark first that $\mathfrak{E} \cap E_{t}$ is empty. Indeed, suppose that $\left(\mathbb{E} \cap E_{t}\right.$ is not empty and let $\phi\left(e^{i \theta}\right)=e^{i \theta_{1}} \in\left(E \cap E_{t}\right.$. If we denote by $L$ the image under $t=\phi(z)$ of

3) See [3] or [10].

4. The author is grateful to Professor Kiyoshi Noshiro who suggested a simplification for the proof of this theorem. 
the radius with end point $e^{\imath n}$, then by Jenkins' theorem $[2 ;$ p. 344$], L$ is a Stoltz path terminating at $e^{i n_{n}}$, along which the cluster set of $w=F(t)$ must lie on the circumference $|w|=1$. On the other hand, we have that $\lim F(t)$

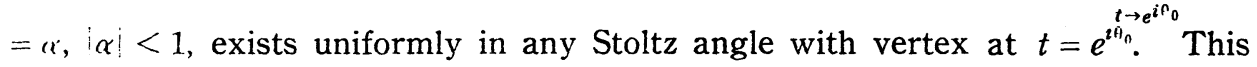
is a contradiction. Thus we have $\left(\xi \subset \widetilde{E}_{t}\right.$. Accordingly, $E$ is of capacity zero and so of linear measure zero. Hence $w=F(t)$ is a function of class $(A)$. Applying a well known theorem of Nevanlinna-Frostman, the theorem is proved.

\section{BIBLIOGRAPHY}

[1] F. Bagemihl, Curvilinear cluster sets of arbitrary functions, Proc. Nat. Acad. Sci. (U.S.A.), 41, no. 6 (1955), pp. 379-382.

[2] J. A. Jenkins, On Quasiconformal mappings, Journal of Rational Mechanics and Analysis, 5 (1956), pp. 343-352.

[ 3 ] A. J. Lohwater, The boundary values of a class of meromorphic functions, Duke Math. Journal, 19 (1952), pp. 243-252.

[4] A. J. Lohwater, Les valeurs asymptotiques de quelques fonctions méromorphes dans le cercle-unité, C. R. Acad. Sci. Paris, 237 (1953), pp. 16-18.

[5] A. J. Lohwater, The reflection principle and the distribution of values of functions defined in a circle, Ann. Acad. Sci. Fennicae A. I., 229 (1956), pp. 1-18.

[6] R. Nevanlinna, Eindeutige analytische Funktionen, 2nd ed. Berlin (1953).

[7] K. Noshiro, $A$ theorem on the cluster sets of pseudo analytic functions, Nagoya Math. Journal, I (1950), pp. 83-89.

[ 8 ] K. Noshiro, On the theory of cluster sets of analytic functions, Sugaku, 5, no. 2 (1953), pp. 65-72 (Japanese).

[9] A. Pfluger, Extremallängen und Kapazität, Comment. Math. Helv., 29 (1955), 120-131.

[10] W. Seidel, On the distribution of values of bounded analytic functions, Trans. Amer. Math. Soc., 36 (1934), 201-226.

[11] S. Stoïlow, Leçons sur les principes topologiques de la théorie des fonctions analytiques, Paris (1938).

[12] D. A. Storvick, On meromorphic functions of bounded characteristic, Proc. Amer. Math. Soc., 8 (1957), 32-38.

[13] G. T. Whyburn, Analytic Topology, Amer. Math. Soc. Colloq. Pub., 28 (1942).

Department of Mathematics

University of Minnesota

Minneapolis, Minnesota 